\title{
Miranda
}

Revue pluridisciplinaire du monde anglophone /

Multidisciplinary peer-reviewed journal on the English-

speaking world

$20 \mid 2020$

Staging American Nights

\section{Nuits intimes et inimitiés nocturnes}

\section{Alain Montandon}

\section{OpenEdition}

\section{Journals}

Édition électronique

URL : http://journals.openedition.org/miranda/24239

DOI : 10.4000/miranda.24239

ISSN : 2108-6559

Éditeur

Université Toulouse - Jean Jaurès

Référence électronique

Alain Montandon, « Nuits intimes et inimitiés nocturnes », Miranda [En ligne], 20 | 2020, mis en ligne le 24 mars 2020, consulté le 16 février 2021. URL : http://journals.openedition.org/miranda/24239 ; DOI https://doi.org/10.4000/miranda.24239

Ce document a été généré automatiquement le 16 février 2021.

\section{c) (i)}

Miranda is licensed under a Creative Commons Attribution-NonCommercial-NoDerivatives 4.0 International License. 


\title{
Nuits intimes et inimitiés nocturnes
}

\author{
Alain Montandon
}

Réfléchir sur la nuit et l'intime pose immédiatement la question de savoir pourquoi la nuit peut être associée à cette notion. Sans doute des clichés s'imposent-ils d'emblée, ceux de la nuit maternelle, réconfortante, douce et chaleureuse, moment du repli, du repos, du sommeil, de la régression en enfance apportée par le sentiment océanique d'une heureuse fusion, douce nuit, sainte nuit, tout est calme, rien ne bruit. Mais que signifie cette intimité ? Sans doute John Field, en inventant la forme musicale du nocturne, y a-t-il associé la subjectivité et une profonde émotion comme le rappelait Liszt en $1859^{1}$. Le piano murmure et chante sans parole, la parole étant remplacée, refoulée par la musique, par l'inexprimable, de ce qui ne veut pas se dire, de la rêverie muette, pudique, du désir à peine entrevu, effleuré, vapeur légère que les phrases simples, reprises, répétées, variées, retrouvées, insistantes et troubles dessinent. On ne saurait analyser le charme de cette spontanéité, de ce jeu léger d'une rêverie dans laquelle on se perd, évocatrice d'un silence et d'une douceur sans effort. Selon Liszt, personne n'a su retrouver après lui un tel langage de l'inexprimable, plein de caresses et de bercements, comme le va-et-vient régulier d'une barque qui se balance ou du mouvement d'un hamac. On assiste à la mise en place d'une musique de l'intimité et de l'évasion, créant une ambiance de nostalgie, de paresse langoureuse et de féminité si l'on en croit Paul Bourget dans ses Nouveaux essais de psychologie contemporaine (1885). Voilà bien l'image d'une intimité domestique, du retrait qu'impose la nuit pour se réfugier dans la maison protectrice et pour se replier sur soi dans une amicale familiarité avec soi-même. «Entends, ma chère, entends la douce Nuit qui marche. ${ }^{2}$ »

2 «Tendre est la nuit » écrivait Keats dans son Ode à un rossignol, vers repris par Francis Scott Fitzgerald pour intituler un de ses romans (1934). Celle que Hölderlin appelle " die Schwämerische », la rêveuse, mais aussi l'essaimeuse (car schwärmen c'est aussi essaimer), car elle est un réservoir inépuisable d'amour, de mystère, de rêverie, de mélancolie, de tendresse et de nostalgie, la nuit, a trouvé dans le chant du rossignol sa plus belle illustration. Théophile Gautier en donne dans son roman Mlle de Maupin une vibrante illustration.

Le ciel, quoiqu'il fit tout à fait nuit, avait une clarté presque égale à celle du plus

beau jour; il était si profond et si transparent que le regard pénétrait aisément 
jusqu'à Dieu. [...] Je ne pensais pas, je ne rêvais pas, j'étais confondu avec la nature qui m'environnait, je me sentais frissonner avec le feuillage, miroiter avec l'eau, reluire avec le rayon, m'épanouir avec la fleur; je n'étais pas plus moi que l'arbre, l'eau ou la belle-de-nuit. J'étais tout cela, et je ne crois pas qu'il soit possible d'être plus absent de soi-même que je l'étais à cet instant-là. Tout à coup, comme s'il allait arriver quelque chose d'extraordinaire, la feuille s'arrêta au bout de la branche, la goutte d'eau de la fontaine resta suspendue en l'air et n'acheva pas de tomber. Le filet d'argent, parti du bord de la lune, demeura en chemin : mon cœur seul battait avec une telle sonorité qu'il me semblait remplir de bruit tout ce grand espace. - Mon cœur cessa de battre, et il se fit un tel silence que l'on eût entendu pousser l'herbe et prononcer un mot tout bas à deux cents lieues. Alors le rossignol, qui probablement n'attendait que cet instant pour commencer à chanter, fit jaillir de son petit gosier une note tellement aiguë et éclatante, que je l'entendis par la poitrine autant que par les oreilles. Le son se répandit subitement dans ce ciel cristallin, vide de bruits, et $\mathrm{y}$ fit une atmosphère harmonieuse, où les autres notes qui le suivirent voltigeaient en battant des ailes. - Je comprenais parfaitement ce qu'il disait, comme si j'eusse eu le secret du langage des oiseaux. C'était l'histoire des amours que je n'ai pas eues que chantait ce rossignol. [...] Il me disait ce que je n'avais pas pu me dire, il m'expliquait ce que je n'avais pu comprendre; il donnait une voix à ma rêverie, et faisait répondre le fantôme jusqu'alors muet. Je savais que j'étais aimé, et la roulade la plus langoureusement filée m'apprenait que je serais heureux bientôt. Il me semblait voir à travers les trilles de son chant et sous la pluie de notes s'étendre vers moi, dans un rayon de lune, les bras blancs de ma bienaimée. Elle s'élevait lentement avec le parfum du cœur d'une large rose à cent feuilles. $»^{3}$

3 Ici l'intime, l'intime plus intime, touche les zones les plus profondes de l'être, des régions non connues, inconscientes, le « ce que je n'avais pas pu me dire », le «ce que je n'avais pu comprendre ». Autrement dit le secret, le plus profond, l'inexprimé qui constitue la profonde identité du sujet. Cette révélation pourrait être commentée avec un tableau de Caspar David Friedrich Homme et femme contemplant la lune $e^{4}$ particulièrement significatif du lien intime de l'être humain et de la nuit.

Donnant par le clair-obscur des états crépusculaires la grandeur aux choses simples et un sens sublime à la nature, Friedrich partage la pensée de Novalis : "Tout devient poésie dans l'éloignement: des montagnes lointaines, des hommes lointains, des événements lointains... Tout devient romantique. De là notre nature primitivement poétique. Poésie de la nuit et du crépuscule ${ }^{5} »$. La profondeur lumineuse de ces nocturnes (Friedrich met l'accent non sur l'obscurité nocturne, mais sur la clarté nocturne) transfigure le paysage en rendant proche le lointain et le lointain proche. L'intime s'enracine, prend corps avec ces personnages vus de dos, hypnotisés par la lumière lunaire, lumière surnaturelle, image de l'âme du monde, symbole d'une totalité, et surtout d'une autre lumière, christique celle-là, comme le sait tout lecteur de Novalis.

5 La présence du personnage sert à l'identification du spectateur, entraîné ainsi dans le tableau, le personnage vu de dos servant en quelque sorte de relais à l'intérieur de l'image, mettant en évidence la présence d'un sujet, d'une subjectivité en communion avec le paysage nocturne. Plus qu'une intégration du regard, de ces yeux que nous ne voyons pas, c'est une intégration du corps tout entier de l'homme dans cette expérience de la nature. Expérience intime et magnétique révélant l'identité profonde de l'homme et de la nature, du sujet et de l'objet, du Moi et du non-Moi, pour reprendre la terminologie de Fichte étudiée par Novalis. 
6 Il y a une harmonie entre le microcosme humain et l'âme du monde (Weltseele). Henrik Steffens, un élève de Schelling, parle d'ailleurs d'un parallélisme entre l'extériorité de la nature et l'intériorité de l'esprit. Ce dialogue entre l'image objective et son intériorisation subjective (par le sentiment, la nostalgie et la rêverie) sert à la prise de conscience de l'identité profonde de l'homme et de la nature et à une rêverie fondamentale, celle d'une aspiration à retrouver l'unité perdue.

7 On voit à ces exemples que l'intime est une relation et une affinité, tout comme il est ce qu'il y a de plus enfoui, de plus secret, de plus réservé. Jullien ${ }^{6}$ a pu, reprenant des définitions anciennes, opposer ces deux conceptions, l'une étant celle d'un intérieur qui se dérobe et l'autre étant son contraire dans la relation à l'autre, le dehors devenant dedans et inversement. L'approfondissement du dedans et l'ouverture au-dehors, loin d'être incompatibles peuvent s'engendrer l'un l'autre.

8 «Still wird die erleuchtete Gasse ${ }^{7}$ » écrit Hölderlin dans la première strophe de Brod und Wein évoquant les grondements des voitures qui s'éloignent alors que descend la paix du soir. À ce retrait de la vie diurne devenue silencieuse s'ajoute le sentiment de la profondeur et d'un espace qui s'accroît, car des silences s'enflent les échos que porte «l'air assombri » (in dämmriger Luft). Tandis que les bruits du jour s'apaisent lentement, d'autres sons s'éveillent au sein du monde devenu silencieux. Le silence se met à bruire sous le souffle impalpable du vent du soir comme animant une harpe éolienne. Les sentiments s'épanouissent, le monde intérieur se dilate et s'ouvre :

Aber das Saitenspiel tönt fern aus Gärten; vielleicht, daß

Dort ein Liebendes spielt oder ein einsamer Mann

Mais des accords résonnent au loin depuis les jardins ; peut-être

Là-bas, est-ce un amant qui joue, ou quelque solitaire

9 La finesse et la délicatesse de ce moment sont signifiées par les jardins qui sont au loin, sans doute à l'extérieur de la ville et qui apportent leurs effluves dans les rues désertées, et aussi par ce "peut-être " qui marque l'indécision dans ses plus fines vibrations. Une nostalgie, bien étrangère à la matérialité objective des préoccupations diurnes, prend son essor, comme le rêve d'un autre monde. Moment d'intériorité qui est moment du souvenir, souvenir de l'amour, de l'amitié, de la jeunesse dans le concert d'une nature sensuelle, fraîche, vivante et parfumée mais que dominent les battements du temps marqué par le son des cloches et la voix du veilleur de nuit qui proclame le compte des heures. Par un bel oxymore, c'est en silence que résonne dans l'air obscur le tintement de la cloche ("Still in dämmriger Luft ertönen [... $\left.]^{8} »\right)$ Ainsi s'instaure le moment musical de la nuit. L'air assombri est d'une plus grande substance, il est épaissi, plus dense, plus charnel.

Novalis quant à lui a encore plus approfondi ce moment d'intériorité dans ses Hymnes à la nuit. L'initiation au mystère de la nuit prend son essor dans le premier Hymne, au sein même de la lumière, non pas dans la monstration de sa pauvreté, mais dans celle de son excès de richesse. L'homme est, par l'intermédiaire de ses sens, touché par la merveilleuse diversité des apparitions phénoménales, mais reste un étranger au milieu de cet univers bariolé. Le sentiment de sa déréliction provient de son extériorité sensorielle, la perception divisant la nature terrestre en de multiples images. C'est l'absence de lumière qui le ramène à l'unité de son être, lui révèle alors son exil vécu dans la dispersion. 


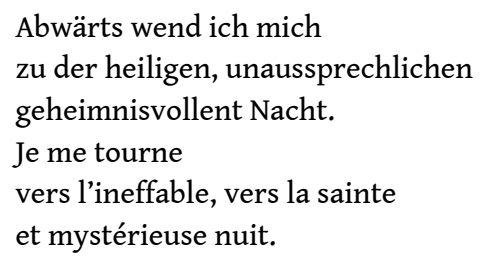

11 Le moi opère une conversion radicale, un détour qui est retour sur lui-même, une descente au fond de soi qui ouvre sur le lien à l'extérieur et à l'autre. La conversion au nocturne commence donc par une conversion vers l'intérieur: "se détacher de l'extérieur, c'est la source de tout abaissement, en même temps que la base de toute véritable élévation ${ }^{9}$. »

12 Alors que le monde diurne peut être saisi et décrit dans le kaléidoscope de ses manifestations, la nuit qui s'installe, installe l'ineffable. L'essai de dénomination apophatique (« unaussprechlich», «inexprimable») nous fait entrer dans le vocabulaire $\mathrm{du}$ «sans limite ». Les ailes de l'âme se déploient et ainsi s'ouvrent les paupières de ces yeux infinis que la nuit ouvre en nous.

«... die unendlichen Augen,

Die die Nacht

In uns geöffnet. » (Hymne I).

13 Ce que la nuit donne à voir, par la métaphore des yeux spirituels, opposés aux cinq sens, c'est l'unité du monde. La nuit est l'expérience intime d'une réintégration avec soi et avec le monde et l'image d'une liquidité de la nuit se retrouve, chez nombre d'écrivains romantiques où elle est employée, à désigner cette union, celle du conscient et de l'inconscient et d'une fusion harmonieuse avec le cosmos.

Mais ce n'est pas toujours le cas, car la nuit intime peut rimer avec abyme ${ }^{10}$.

Les représentations de la nuit sont tributaires des époques historiques, des valeurs culturelles, sociales et religieuses. Ainsi la nuit, privation de lumière, a-t-elle été dans le passé le moment des peurs et des angoisses, avec leurs fantômes et apparitions que la critique rationnelle s'efforce de chasser, mais aussi l'image de la superstition et de l'obscurantisme. Nombre d'écrivains des Lumières ont encore une vision archaïque de la nuit : c'est le domaine des forces du Mal. La nuit était considérée comme un espacetemps échappant aux lois ordinaires. Joachim Schlör a rappelé avec raison dans son ouvrage $^{11}$ que les lois juridiques pouvaient être autrefois différentes de nuit, la nuit exigeant une appréhension différente des troubles survenus. C'est que - pour les actants nocturnes eux aussi -les règles et les normes s'estompent, disparaissent ou se modifient. La nuit est le repaire du crime ${ }^{12}$, tout comme elle est l'image même de ce sommeil de la raison qui engendre des monstres, ainsi que Goya a pu le dépeindre ou encore Füssli dans ses tableaux du cauchemar. La bipolarité traditionnelle de la lumière et des ténèbres, conçue comme l'opposition du Bien et du Mal, de Dieu lumière et d'un diable enténébré subsiste.

L'évolution technique et culturelle vient bouleverser à partir de la fin du XVIII ${ }^{\mathrm{e}}$ siècle les rapports de l'homme à la nuit. Les grands progrès de l'éclairage contribuent à modifier les rapports de l'homme à l'obscurité. Ceci semble avoir pour conséquence un regain d'attrait pour la nuit, la source des rêves et la psychologie empirique comme les spéculations des sciences de la nature y trouvent un nouveau champ d'investigation. Ce qui se passe la nuit devient particulièrement digne d'intérêt. Voir la nuit, écrire la nuit deviennent des enjeux majeurs, évidemment paradoxaux dans la mesure où la nuit semble soustraire tout objet à la vision et où l'écriture de la nuit semble relever d'une 
gageure, car avec la nuit nous entrons dans une crise de la représentation qui est une des sources fondamentales de la modernité. Avec les objets nocturnes est posé le problème de l'irreprésentable. Ce qui caractérise la nuit est bien le fait que la vision s'y égare, qu'elle échappe, fuit, s'annihile, c'est-à-dire que se pose le problème de l'irreprésentable et avec lui une crise profonde de la mimesis qui avait été jusqu'alors au fondement de l'esthétique et que l'on assiste à un changement de paradigme. Du coup la nuit devient un terrain privilégié des récits fantastiques (depuis Hoffmann, Edgar Poe et chez leurs nombreux imitateurs).

En outre, cette révolution des éclairages a sans aucun doute fait naître une nostalgie profonde à l'idée que quelque chose va irrémédiablement se perdre dans un monde où «le gaz a remplacé le soleil» comme l'écrivait Jules Janin en $1839^{13}$. Lorsque les noctambules parisiens parcourent la cité de nuit, ils le font avec le sentiment que c'est toute une ville qui va disparaittre sous la lumière. Simone Delattre se demande non sans raison, dans son riche livre sur Les douze heures noires, si les Noctambules des années 1840 ne cherchent pas «à fixer pas à pas le souvenir d'un Paris ombreux qu'on sent en voie de disparition ${ }^{14}$ "

18 Avec le $\mathrm{XIX}^{\mathrm{e}}$ siècle et particulièrement depuis Baudelaire et le naturalisme, l'emprise de la ville sur l'homme est devenue patente, ce que confirmera la sociologie urbaine de l'école de Chicago. La ville et la nuit, toutes deux, imposent leur domination sur la vie et le comportement des hommes. Avec l'industrialisation et l'urbanisation croissante c'est une vie artificielle qui se fait jour, avec de nouveaux éclairages qui apportent de nouvelles couleurs et de bien étranges nuances. Bien plus que le jour, la nuit absorbe les humains dans l'espace d'une foule indistincte et anonyme. Le flâneur noctambule s'y confond et s'y noie, comme cet homme des foules auquel Edgar Allan Poe a donné, en 1840, un visage (The Man of the Crowd), un homme qui ne supporte pas d'être seul parce qu'il porte en lui quelque secret. La fascination de se mêler anonymement aux autres et l'angoisse de s'y perdre témoigne de l'ascendance du phénomène nocturne, qui meut l'être par des impulsions qu'il ne contrôle pas, comme dans le somnambulisme et l'hypnotisme. Autrement dit, la nuit impose un nouveau registre, un mode d'être non rationnel, invitant à des régressions et d'archaïques retours. La nuit renvoyant l'homme à lui-même génère des troubles identitaires.

Avec les nouveaux éclairages, c'est la dernière frontière qui disparait progressivement avec l'empiétement des activités diurnes sur la nuit. C'est toute une économie festive qui apparaît avec les bals, les théâtres, les bars, les dancings, les cafés, etc.

Pas une ville au monde n'offre le spectacle de ces boulevards parisiens surtout à certaines heures. Le soir, quand le gaz s'allume, quand théâtres, cafés-concerts, grands bazars, estaminets dorés ou pauvres, allument leurs enseignes et leurs candélabres, quand les fenêtres des grands cercles flambent, quand sur le pavé les traînées d'électricité font comme des rivières d'argent, qui parlera des a giorno de Venise et des illuminations de l'Orient !15

Mais on pourrait dire la même chose de toutes les grandes villes au $\mathrm{XX}^{\mathrm{e}}$ siècle, vérifiant ainsi la célèbre constatation de Baudelaire suivant laquelle «la forme d'une ville/ Change plus vite, hélas! que le cœur d'un mortel ${ }^{16}$ ». La nuit représente sans conteste le comble de l'artifice avec les cafés, les boulevards qui «flamboient », un mot qui revient sans cesse sous la plume des écrivains avec l'image des incendies de lumière qui s'allument à la tombée du jour. Les sirènes des voitures de pompier traversant la ville nocturne donnent un relief sonore au Manhattan de Dos Passos. 
21 L'esthétisation des choses par la lumière artificielle crée un monde bariolé, plein de dérèglement, de flux et de mouvement, un émerveillement aux nombreuses ambiguïtés. L'alternance de l'ombre et des lumières n'est pas sans évoquer une féerie factice, un enchantement de pacotille. "Les filles surtout, avec la traîne de leur robe, tour à tour crûment éclairées et noyées dans l'ombre, prenaient un air d'apparition, de marionnettes blafardes, traversant le rayon électrique de quelque féerie " écrit Zola dans La Curée $e^{17}$. C'est que la nuit métamorphose les êtres et donne un lieu aux êtres de la nuit que sont les fêtards, les mendiants, les prostituées, les ivrognes, les chiffonniers, les chiens errants, les vagabonds et les clochards, tous êtres marginaux, car la nuit est l'espace marginal du jour.

La nuit révèle le fond inconscient, tout le côté obscur et refoulé de l'âme humaine. Ainsi de Mister Hyde et du docteur Jekyll, dont l'un est un être de nuit, tandis que l'autre est un être du jour dans l'opposition entre l'homme criminel et l'homme normal. La mise en scène des perspectives nocturnes comme dans le Cabinet du docteur Cagliari de Wiene crée une ambiance d'angoisse avec les angles obliques des maisons se penchant sur des ruelles tortueuses. Comme le dit un critique: "Les lampadaires ivres annoncent l'hallucination véhémente d'un crime prêt à se commettre ». Le cinéma n'est pas en reste comme le montre une image de M. le maudit (1931) où Fritz Lang offre la vue d'un univers minéral pétrifié où les humains apparaissent comme des témoins menaçant ou d'atroces victimes potentielles. On comprend alors le mécanisme des répétitions et du délire obsessionnel chez M. le maudit dont le démon s'éveille la nuit :

Toujours, je dois aller par les rues, et toujours je sens qu'il y a quelqu'un derrière moi. Et c'est moi-même! [...] quelquefois c'est pour moi comme si je courais moimême derrière moi! Je veux me fuir moi-même mais je n'y arrive pas! Je ne peux pas m'échapper! [...] quand je fais ça, je ne sais plus rien... Ensuite je me retrouve devant une affiche et je lis ce que j'ai fait, et je lis. J'ai fait cela?

Edward Hopper reprend ce thème où la monumentalité des bâtiments écrase dans une perspective foudroyante l'homme de la rue dans Ombres nocturnes, une eau-forte de 1921. La perspective en hauteur et à l'oblique qui rompt les parallèles par l'introduction de diagonales donne à la vue excentrique et les ombres qu'elle produit un aspect inquiétant.

24 La nuit exacerbe le côté sauvage de la ville qui apparaît comme une espèce de surnature et nombreuses sont les images qui renvoient à celles extrêmes d'une nature sauvage, barbare, cruelle, inhospitalière : les vagues de l'océan, les solitudes minérales du désert, la jungle épaisse, ses broussailles impénétrables, tout parle d'une puissance excessive qui plonge la civilisation dans les ténèbres de l'origine. Là, la civilisation s'arrête, les repères de la culture s'évanouissent, la bouche d'ombre ouvre grand sa gueule pour engloutir dans son cauchemar le promeneur téméraire et imprudent.

Aussi la ville se fait paysage d'une nouvelle nature et l'extrême modernité se voile des accents les plus originels. La ville est une nouvelle jungle et les éléments si caractéristiques du décor urbain appellent chez le flâneur des images d'espaces sauvages. La cour des Tuileries apparaît comme une «immense savane plantée de becs de gaz au lieu de bananiers $»^{18}$. Le candélabre " ressemble à un cocotier au milieu d'une savane ${ }^{19}$. La présence de ces Mohicans de Paris (titre d'un ouvrage d'A. Dumas) rend possible tout un imaginaire du roman populaire comme du roman policier ${ }^{20}$. Les conflits quotidiens de la civilisation ne sont-ils pas semblables à ceux de l'état sauvage? Georges Banu ${ }^{21}$ voit dans cette nuit fantastique à la fois la nuit des villes et la nuit des 
hommes, la ruine et la frayeur, la menace policière pouvant envahir les espaces privés ou encore la nuit du totalitarisme.

L'usage même des espaces, publics ou semi-publics, conforte le sentiment de liberté ou d'angoisse que l'investissement affectif et imaginaire de la nuit surdétermine. La littérature et le cinéma ont amplifié à souhait la représentation de la dimension paranoïaque que pouvaient prendre les nuits urbaines. Il suffit de songer par exemple aux films de Martin Scorsese, de Prévert et Marcel Carné (Les Portes de la Nuit, 1946) ou encore à la nouvelle de Maupassant "La Nuit, Un cauchemar». Le flâneur de Maupassant ne fait pas mystère de l'attachement passionné et libidinal qu'il entretient avec la nuit ${ }^{22}$ :

J'aime la nuit avec passion. Je l'aime comme on aime son pays ou sa maitresse, d'un amour instinctif, profond, invincible. Je l'aime avec tous mes sens, avec mes yeux qui la voient, avec mon odorat qui la respire, avec mes oreilles qui en écoutent le silence, avec toute ma chair que les ténèbres caressent ${ }^{23}$.

$\mathrm{Au}$ jour bruyant et fatigant, monotone et pesant, il préfère la tombée de la nuit et l'ivresse de la noire immensité qui s'approche et le ranime par cette espèce de volonté destructrice. Ainsi parle-t-il de la grande ombre douce tombée du ciel qui « noie la ville, comme une onde insaisissable et impénétrable, elle cache, efface, détruit les couleurs, les formes, étreint les maisons, les êtres, les monuments de son imperceptible toucher $^{24}$ ». La nuit est assimilée à l'élément liquide, élément séducteur au charme profond et traître. Une irrésistible envie de baigner dans l'eau de la nuit, de courir à travers les rues le saisit, un invincible désir de rôder comme "mes sœurs les bêtes et mes frères les braconniers ", du côté des révoltés et des marginaux.

Peut-être y a-t-il chez Maupassant une réminiscence du Spleen de Paris quand Baudelaire évoquait le soulagement qu'apporte la nuit à la vie de la capitale : « Ô nuit ! ô rafraîchissantes ténèbres! vous êtes pour moi le signal d'une fête intérieure, vous êtes la délivrance d'une angoisse! Dans la solitude des plaines, dans les labyrinthes pierreux d'une capitale, scintillement des étoiles, explosion des lanternes, vous êtes le feu d'artifice de la déesse Liberté $! »^{25}$ Baudelaire évoque par ailleurs les hiboux ${ }^{26}$ et les sinistres ululations de la ville nocturne («Le crépuscule excite les fous»). Maupassant quant à lui montre comment la plongée dans les ténèbres est contemporaine de l'obscurcissement de l'esprit.

Un sentiment nouveau de froid et de pesanteur oppressante accompagne l'avenue désormais déserte sous l'éclairage des becs de gaz «qui paraissaient mourants" (comme si cette asthénie lumineuse présageait sa propre mort). «Je n'avais jamais vu Paris aussi mort, aussi désert. Je tirai ma montre, il était deux heures..$^{27} »$ Aussi se rendil à la Bastille.

Là, je m'aperçus que je n'avais jamais vu une nuit si sombre, car je ne distinguais pas même la colonne de juillet, dont le Génie d'or était perdu dans l'impénétrable obscurité Une voûte de nuages, épaisse comme l'immensité, avait noyé les étoiles, et semblait s'abaisser sur la terre pour l'anéantir ${ }^{28}$.

Il ne peut entrer en communication avec les rares personnes rencontrées (un ivrogne, une prostituée, un fiacre, un chiffonnier). C'est alors qu'il s'aperçoit que les becs de gaz étaient éteints (une coutume assez fréquente encore à l'époque, lorsque la nuit n'est pas trop sombre ou que celle-ci est bien avancée). Continuant à s'avancer, à l'aveuglette dans ces « rues solitaires et noires, noires, noires comme la mort » il se perd dans une obscurité et un silence qui semblent anormaux et quasiment fantastique. Le son même de sa propre voix est étouffé par cet air épaissi de l'ombre. «J'appelai plus fort. Ma voix 
s'envola, sans écho, faible, étouffé, écrasé par la nuit, par cette nuit impénétrable. » Ses appels au secours sont sans réponse et l'errance continue avec ses souffrances physiques et psychiques. Il a beau frapper aux portes, ni les concierges ni les habitants ne répondent. Arrivé aux Halles (où il avait vu se diriger maintes voitures de légumes chargées de carottes, de navets et de choux), à ces mêmes Halles où il pensait trouver la vie, il découvre qu'elles sont « désertes, sans un bruit, sans un mouvement, sans une voiture, sans un homme, sans une botte de légumes ou de fleurs. - Elles étaient vides, immobiles, abandonnées, mortes ${ }^{29}$ ! L'épouvante le saisit devant ce qui semble être la fin du monde, sa montre elle-même est arrêtée (comme dans la gravure de Hogarth signifiant la fin des temps et comme dans de nombreuses descriptions fantastiques de la nuit qui, par la suspension même du temps qu'elle suggère, multiplie les montres sans aiguilles et les montres sans cadran). La logique le conduit alors à sa propre mort en descendant dans la Seine, dans l'eau froide du fleuve parisien, une eau «froide... presque gelée... presque tarie... presque morte ", liquidité funèbre et mortifère, une eau morte comme celle que l'on trouve chez Edgar Allan Poe. Marie-Claire Bancquart qui parle, à propos de cette expérience nocturne de vampirisation de soi-même écrit qu'ici «l'autisme noir a triomphé $»^{30}$.

31 Catherine Deschamps a pu remarquer que dans les séries télévisuelles ou dans des romans des meurtres, la nuit parait être «le paysage de prédilection pour la mise en scène d'agressions, assassinats ou viols dont les femmes sont victimes en extérieur ou dans les parkings souterrains - ces lieux couverts fictionnels et fantasmatiques qui, à l'instar des rues nocturnes, ne sont éclairés que par des lumières artificielles et faméliques. Dès lors, si ce n'est dans les faits du moins dans leur scénarisation, les nuits apparaissent comme facteur de surdétermination des risques pour les femmes ${ }^{31} »$. Les films noirs ont tout particulièrement ravivé angoisses et désirs de transgression.

La nuit, comme le sommeil de la raison, engendre des monstres. Cette peur archaïque de l'inconnu, trouble lié à la séparation vespérale, est une phobie signalée par Freud "Quant à la solitude, au silence et à l'obscurité, [...] ce sont là effectivement les circonstances auxquelles s'attache chez la plupart des humains une angoisse infantile qui ne s'éteint jamais tout à fait ${ }^{32}$ ». La peur du noir, peur de l'obscurité, a été immortalisée par Proust, le «drame du coucher » renouant la douleur de la séparation et la perte de la chaleur familiale et de la présence maternelle. Lorsque les ombres de la lanterne magique accentuent les effets conjugués de l'angoisse et de l'obscurité par la mise en scène de personnages agressifs et que le jeune garçon revêt «le suaire de [s]a chemise de nuit ${ }^{33}$ ", il entre alors dans un monde d'une inquiétante familiarité, celui du monde dissolvant des ténèbres. La nuit se fait liquide, eau noire, "substance symbolique de la mort ${ }^{34} »$. Dans la nuit, les sensations s'exacerbent, les sons prennent une dimension inquiétante, les objets prennent un aspect insolite et l'angoisse grandit d'autant plus qu'elle est innommable. L'inanimé s'anime, le monde s'animalise et se peuple d'un bestiaire nocturne effrayant. «Les terreurs de la nuit mettent en jeu de véritables mécanismes autant physiologiques, psychologiques que culturels ${ }^{35} »$ comme l'a montré entre autres Alain Cabantous, dont on trouve l'écho non seulement dans les albums pour enfants comme l'a montré Christiane Connan-Pintado dans son article du Dictionnaire littéraire de la nuit ${ }^{36}$, mais de manière plus générale dans de nombreux textes de la littérature.

33 L'entrée dans la nuit, par la perte des repères, favorise une régression du sujet dans l'enfance, dans le surgissement de souvenirs à la fois personnels, mais aussi souvenirs 
de schèmes culturels archaïques, celui des contes quand ce n'est pas une dimension mythique ou mythologique qui se trouve réactivée comme on peut le voir de manière exemplaire dans la nouvelle de Truman Capote intitulée L'Arbre de nuit publiée d'abord en octobre 1945 dans Harper's Bazaar, puis en volume en 1949.

L'écrivain donne d'emblée l'atmosphère, celle d'un monde nocturne dépourvu de chaleur. "C'était l'hiver. Une rangée d'ampoules électriques nues, qui semblaient vidées de toute chaleur, illuminait le quai de la petite gare, où soufflait une bise glaciale $^{37} »$. Cette mise en scène symbolique, celle du départ d'un train dans une gare, sur un quai isolé, un soir d'hiver est décrite par des images métaphoriques angoissantes, puisqu'il compare les glaçons qui pendent aux gouttières à des «dents menaçantes de quelque monstre de cristal ». Le décor est planté où la jeune fille attend le train dans un quai où ne se trouve âme qui vive. L'arrivée du train comporte aussi dans sa description quelque chose de monstrueux, lui qui arrive tel un dragon, « giclant de vapeur et éblouissant de lumière » dans un fracas de tonnerre. Si j'insiste sur ces éléments, c'est que dès le départ la nouvelle peut être lue doublement. Elle est en effet d'une part le récit de l'aventure très réelle d'une jeune fille un peu perdue et désemparée par la solitude nocturne qui prend place dans un wagon assez délabré en compagnie de personnages sinistres qui finiront par avoir raison de son innocence et de sa naïveté en la dépouillant et peut-être même - le récit se termine de manière ambiguë - en l'assassinant, la femme rabattant à la fin du récit l'imperméable sur sa tête "comme un linceul ». Mais, d'autre part, le récit prend dès le départ un aspect onirique indiquant par les images évoquées que le lecteur épouse la vision de la jeune fille angoissée par ce voyage nocturne, et dont le désemparement amène à percevoir ce qui l'entoure de manière effrayante et fantastique au point même que réalité et irréel se confondent et que conscience et cauchemar se mêlent dans une sorte de rêve éveillé qui finira dans un véritable sommeil à la fin, quand elle se laisse aller « dans une douce somnolence ".

35 Le train dans lequel elle monte ressemble plus dans cette nuit obscure à un train fantôme, car on ne sait ni d'où il vient ni où il va ${ }^{38}$. La perte des repères géographiques est d'ailleurs totale et à la question de la passagère qui lui demande d'où la jeune fille vient et où elle se rend, la réponse est plus qu'évasive puisque la gare de ce trou perdu est loin d'une ville et qu'elle-même n'y habite pas puisqu'elle avait été simplement là pour assister aux funérailles d'un oncle et qu'elle retournait à l'université (indice pouvant lui donner un âge). À l'insistante question, la jeune fille est déroutée et n'arrive pas à trouver de réponse : « les noms de plusieurs villes lui vinrent tout à coup à l'esprit. Finalement de ce chaos elle réussit à extraire : - La Nouvelle-Orléans. J'habite La Nouvelle-Orléans. »

36 La jeune fille est prénommée Kay et l'on ne peut qu'être sensible au fait que le nom se rapproche aussi bien du français « quai » (Truman Capote connaît bien le français dont il use parfois dans ses écrits), mais surtout de l'anglais «key", la clef, mais la clef de quelle énigme? À moins qu'elle ne soit à elle-même sa propre clef dans ce parcours témoignant, que l'énigme est en elle. Car ce qui frappe le lecteur est bien l'angoisse grandissante au cœur de la nuit et l'identité instable d'un être déstabilisé oscillant entre réel et irréel, étreint d'une peur maladive de la nuit, amenée à voir surgir du plus profond d'elle-même des souvenirs de l'enfance.

37 La fragilité de la jeune femme est signifiée par l'attitude infantile et timorée dans laquelle elle se réfugie. Son bagage est plus celle d'une adolescente que d'une jeune 
femme : elle a une guitare verte, elle lit des illustrés et son sac porte non son nom mais son prénom. De plus, elle n'acceptera pas de boire le verre de gin qu'on lui offre et le versera subrepticement dans la guitare. Elle a pourtant dix-neuf ans, mais elle pleurnichera comme un enfant colérique. Toute son attitude témoigne de son étonnement envers ce qui l'entoure, voire son effarement devant ce qu'elle observe et découvre. Ainsi s'attache-t-elle à regarder avec curiosité la cigarette de l'homme palpitante «comme la lueur chaude d'une luciole» et les anneaux de fumée qui s'élèvent dans l'air.

38 La nuit l'assaille avec ses bruits effrayants et le tintamarre cacophonique qui l'entoure (" fracas de tonnerre », « sifflement », son des cordes, braillement de la femme, cris de l'enfant à travers son sommeil, cloche qui tinte follement, ronflements, roulis de la bouteille, ronronnement lointain des roues du train, bruit de ferraille du roulement $\mathrm{du}$ train, froissement des journaux, etc.). Elle en est comme anéantie, perdant la voix, comme tétanisée, paralysée dans ce monde en mouvement. Elle est comme une enfant confrontée à l'inquiétante étrangeté du monde des adultes, vivant un cauchemar qui exprime le «malaise profond de la jeune fille encore imprégnée de l'enfance et de ses terreurs ${ }^{39}$ ».

39 Sans doute Truman Capote retrouve-t-il aussi dans cette évocation les terreurs de sa propre enfance, lorsqu'à quatre ans il assiste aux disputes parentales d'une grande violence. Il est aussi laissé seul le soir et la nuit, abandonné dans des chambres d'hôtel, enfermé à clé, terrorisé. Il dira avoir vécu sa petite enfance dans la crainte permanente de l'abandon et il fut d'ailleurs délaissé par ses parents.

Le moment régressif est marqué par le fait qu'elle prend place dans le dernier wagon et le thème de la fin indique que loin d'aller vers l'avenir et l'aurore elle retourne vers le passé. Elle ne trouve qu'une petite place dans « un compartiment isolé », « tout au bout du wagon ", après l'avoir traversé.

C'était plutôt une relique qu'un wagon: tout l'intérieur était délabré; les sièges antiques de peluche rouge étaient par endroits usés jusqu'à la corde et la peinture couleur teinture d'iode des boiseries s'écaillaient. La lampe de cuivre vénérable qui pendait au plafond avait un air romantique et incongru. Une fumée fuligineuse et sinistre flottait dans l'air et l'atmosphère surchauffée du wagon accentuait encore le remugle des sandwiches jetés à même le plancher parmi les trognons de pomme et les écorces d'orange. Cette litière de détritus, qui comprenait également des gobelets de papier, des bouteilles de limonade et des journaux déchirés, jonchait toute la longueur du couloir. ${ }^{40}$

On voit combien les choses témoignent de leur passé d'une part et combien cette évocation est liée à des images répugnantes, repoussantes, nauséabondes et immondes. «Sale nuit!», pourrait-on dire. Et avec une secousse le train s'enfonce dans la nuit, dans l'effacement et la disparition : « un suaire de vapeur blanche frôla la fenêtre avec un sifflement; lentement, les pauvres lumières de la gare solitaire s'estompèrent dans la nuit ». Avec l'image du suaire, l'idée de la mort réapparait, renouant avec celle de l'enterrement de l'oncle. Cet oncle inconnu, dont on ne sait rien, si ce n'est que pour tout héritage, il a laissé à sa nièce une guitare, était quand même une personne qui aurait pu être intime en tant que membre de la famille. Mais il est flagrant que tout lien d'intimité est nié. Les passagers du wagon témoignent soit de l'indifférence envers Kay, soit une forme d'agressivité qui sont autant d'effraction dans la propre sphère intime de la jeune fille: il lui est imposé une conversation qu'elle ne souhaite pas et des « regards scrutateurs ». La nuit intrusive bouscule toute possibilité d'intimité chez Kay 
que le train emporte dans «la poix des ténèbres ». Un parallèle est établi entre la marche du train et le ciel nocturne, avec la " lune d'hiver glaciale [tournant] au-dessus $\mathrm{du}$ train dans le ciel nocturne comme une mince roue blanche ${ }^{41} »$. Dans cette sorte de marche mortelle vers l'enfer, "le ciel était d'un bleu profond inexplorable, peuplé d'étoiles qui s'éteignaient çà et là. Elle apercevait des traînées de fumée qui suivaient dans le sillage de la locomotive comme des coulées d'ectoplasme». Ce dernier mot souligne le caractère fantomatique et irréel de ce que Kay est en train de vivre.

Le couple avec lequel elle partage le compartiment est composé de personnages grotesques, monstrueux, comme échappés d'un cirque malsain. Difformités et infirmités en font des êtres fantastiques et angoissants. Leur grossièreté et leur vulgarité appellent des images d'animalisation (" yeux de mouton ", " cheveux coiffés à la chien ») aux yeux de Kay dont on voit la crainte de la jeune fille d'un niveau social supérieur par son éducation (elle est étudiante à l'universitée ${ }^{42}$ ) envers ces gens plébéien, venant du bas peuple ordinaire, aux allures triviales et vulgaires (impliquant à ses yeux un côté canaille et crapuleux). Ainsi les représentations sociales contribuentelles à noircir sa vision.

Essayant de s'échapper sur la plateforme, elle n'arrive cependant pas à retrouver le calme au sein de cette nuit glaciale ni son identité menacée. Elle se répète où elle se trouve, en Alabama, et "J'ai dix-neuf ans, j'aurai vingt ans au mois d'août et je suis étudiante de deuxième année ». Les étranges personnages surgis d'une nuit d'hiver attisaient des souvenirs imprécis. Du petit homme étrange, elle constatait qu'il y avait en lui « une qualité indéfinissable qu'elle n'arrivait pas à formuler et qui lui rappelait... Quoi? » C'est alors que l'homme du compartiment se glisse sur la plate-forme et en fixant son visage inexpressif, Kay comprend brusquement l'origine de son désarroi.

Kay savait ce qui lui faisait peur : c'était un souvenir, le souvenir des terreurs enfantines qui, jadis, il y avait bien longtemps, planaient au-dessus de sa tête comme les branches hantées d'un arbre de nuit. Tantes, cuisinières, étrangers, tous débitaient à qui mieux mieux des histoires ou enseignaient des chansons, où il était question de fantômes et de mort, de présages, d'esprits, de démons... Et toujours, invariablement, revenait la menace de Croquemitaine : « Reste près de la maison, mon enfant, sinon Croquemitaine t'emportera et te mangera vivante! Il était partout, Croquemitaine, et partout il y avait du danger. La nuit, au lit, ne tape-t-il pas à la fenêtre ? Écoute ${ }^{43}$.

Le monde de la nuit est plein de ces histoires, racontées le soir, contes effrayants d'êtres dévorant les enfants, ogres, Cronos, Saturne. Lorsque l'homme sort de sa poche un noyau de pêche qu'il se met à caresser "de façon vaguement obscène ", il le frotta sur son pantalon pour le faire briller et le plaçant entre ses dents le mordit. Un grand contraste avec le moment précédent où il s'était mis à caresser la joue de la jeune fille. Séduction perverse qui n'est pas sans rappeler Barbe-Bleue à la sensualité mortifère ${ }^{44}$. De même l'arbre des nuits avec ses branches menaçantes fait-il songer à ceux de la ballade de Goethe du Roi des Aulnes (1782) que mettra plus tard en musique d'une belle manière dramatique Schubert en 1813 et ensuite bien d'autres. Là encore, l'espace nocturne est un espace de ravissement et de mort.

Par une nuit très venteuse, le père emmène à cheval son jeune fils, le tenant fermement et le réchauffant (Wer reitet so spät durch Nacht und Wind? Es ist der Vater mit seinem Kind. Er hat den Knaben wohl in dem Arm, Er fasst ihn sicher, er hält ihn warm ${ }^{45}$ ). Il ne fait pas de doute que l'enfant est malade et qu'il croit voir dans son délire le roi des aulnes dans l'obscurité. Il le décrit avec sa couronne et sa trâne. Le père lui explique que ce n'est 
qu'un banc de brume, que les promesses entendues ne sont que le murmure du vent dans les feuilles sèches. Mais la figure royale exerce ses pouvoirs de séduction pour entraîner l'enfant dans son royaume, l'invitant à venir jouer avec lui dans son royaume merveilleux. Le père explique à l'enfant que les formes imaginées des filles du Roi des Aulnes qu'il croit voir ne sont que de vieux saules gris. La vision devient plus menaçante à mesure que monte la fièvre de l'enfant pris de panique devant la figure fantastique :

- Je t'aime, ton joli visage me charme,

Et si tu ne veux pas, j'utiliserai la force.

- Mon père, mon père, maintenant il m'empoigne!

- Le Roi des Aulnes m'a fait mal ${ }^{46}$ !

Le père frémit et presse son cheval, arrive à grand peine, plein d'angoisse, à son port, mais l'enfant était mort dans ses bras (In seinen Armen das Kind war tot.).

Le récit peut être entendu d'une double manière : l'enfant a la fièvre et délire ou bien l'enfant voit réellement l'apparition fantastique et le jugement rationnel du père méconnait alors la réalité de l'emprise de l'être surnaturel. L'hésitation à laquelle la nuit prête pleinement son concours entre ces deux interprétations fonde justement le fantastique tel que l'a défini Todorov. On retrouve cette hésitation chez le lecteur de Truman Capote qui peut donner toute crédibilité à une vision objective du récit ou voir dans certaines exagérations la conséquence de l'angoisse de la jeune fille. Cette suspension du jugement se retrouve à la fin où l'on ne sait si la jeune fille est assassinée ou si elle s'endort tout simplement : «le visage de ce dernier parut changer d'aspect et s'effacer devant elle comme un rocher en forme de lune glissant et s'engloutissant sous une nappe d'eau... Elle se laissa aller à une chaude somnolence et eut vaguement conscience que la femme s'emparait de son sac et qu'elle rabattait doucement l'imperméable sur sa tête comme un linceul ${ }^{47}$. » Était-ce pour l'étouffer, pour lui cacher le larcin en train d'être commis ou bien pour qu'elle ne prenne pas froid dans son sommeil?

Pour revenir au Roi des Aulnes, il est aussi évident que certains vers ont un écho sexuel important. Quels sont les jeux auxquels le Roi des Aulnes veut se livrer avec l'enfant, lui qui lui susurre «Veux-tu, gentil garçon, venir avec moi ? » ou «Je t'aime, ta beauté me charme, et, si tu ne veux pas céder, j'userai de violence ». L'évocation d'une séduction sexuelle et d'un viol («Mon père, mon père, maintenant il m'empoigne! Le Roi des Aulnes m'a fait mal!») sont au cœur de cette sombre histoire. Or il est clair que ces arbres hantés dans la nuit avec leurs bras préhenseurs éveillent chez Kay le souvenir de ce type de récit, avec le trouble généré par l'angoisse sexuelle.

Certains interprètes voient dans le Roi des aulnes l'incarnation des premiers sentiments inconscients de la puberté d'une nature qui apparaît démoniaque à l'enfant privé de son innocence et forcé de quitter son enfance. Le galop du père pour ramener le fils à la maison signifierait une résistance désespérée devant les lois de la nature. Or si nous poursuivons notre parallèle, nous pouvons également comprendre le désarroi de Kay comme son angoisse face à son propre accès au monde de la sexualité. On retrouve les mêmes fantasmes d'agression dans la nouvelle de Truman Capote Monsieur Maléfique ${ }^{48}$ engendrée par la nuit lors d'une promenade dans Central Park.

Le refoulement de la sexualité de Kay permet de mieux comprendre l'attention insistante portée sur la saleté, sur les corps grotesques, mais aussi son ambivalence entre l'image de la caresse et celle de la morsure. Qu'elle soit dégoûtée de voir la femme 
tripoter nerveusement ses boucles, balancer ses jambes dodues, croisées aux chevilles, d'avant en arrière est un fait. Plus curieux, mais combien évocateur sans doute, l'étrange impulsion l'obligeant à s'agenouiller et à toucher la lampe de la plate-forme du train : «Son gracieux tuyau de verre était chaud et la lueur rougeoyante pénétra ses mains et les rendit lumineuses. La chaleur lui dégela les doigts et lui donna des picotements le long des bras. ${ }^{49}$ " Plus tard, elle s'abandonne dans l'image finale à sa jouissance, nouvelle conquête de sa féminité concomitante de la perte, ô combien symbolique, de son sac.

51 Il est banal de voir dans la nuit l'expression du Ça. Mais la subtile écriture de Truman Capote sait faire affleurer différentes strates entre la sordide agression d'une jeune fille dans un train de nuit, la vision onirique et fantasmatique de Kay « faisant naître des doubles monstrueux, reflets de son obscur objet de désir ${ }^{50}$ ", une régression psychique à la dense intertextualité renvoyant au monde des contes, et plus encore aux récits archaïques que sont les mythes, mais traités avec une fine ironie, où Kay serait un Orphée féminin accompagné de sa guitare verte en guise de lyre pour une descente au pays des ombres. Une telle réécriture du mythe tourne vite court, car Kay ne joue pas de son instrument sur lequel elle ne fait que tambouriner vaguement. Le mythe est évoqué pour être immédiatement retourné, tout comme l'histoire de Lazare dont la résurrection n'est évoquée que sous un mode parodique, soulignant de fait qu'aucune résurrection, aucun retour en arrière n'est possible, que la mort est quelque chose de définitif.

Puisque nous étions dans le train, restons dans la vision angoissante de ces transports collectifs d'individus solitaires s'enfonçant dans les ténèbres et regardons comment Edward Hopper évoque l'atmosphère nocturne d'une intimité dans le wagon dans l'eauforte intitulée Night on the El train (1918). À la fois l'espace public de la rame et les ténèbres extérieures renforcent le sentiment d'un renfermement, conférant un caractère presque tragique à l'entretien des deux personnages. À l'inverse de l'eauforte intitulé House Tops de 1921, où la passagère contemple de jour le paysage urbain extérieur qui défile à ses yeux, les fenêtres du wagon, loin de s'ouvrir vers l'espace extérieur, sont aveugles, fermées par une totale obscurité.

De fait, les nuits sont fortement anxiogènes et l'eau forte d'Edward Hopper Ombres nocturnes (Night Shadows, 1921) rend bien, avec une perspective prise en hauteur, plongeante et excentrique qui allonge les ombres, l'aspect imposant, pesant, menaçant de la nuit. Le promeneur nocturne et solitaire est écrasé par ce climat d'angoisse renforcé par la monumentalité des architectures. Une telle vue inspira tant les photographes (Alexandre Rodtchenko ou Otto Umbehr qui rend si mystérieusement étranges les rues) que les cinéastes.

54 Le tableau Nighthawks (1942, 76 x $155 \mathrm{~cm}$ ) qui semble issu d'une vue hollywoodienne panoramique n'est pas sans évoquer les films noirs de gangsters affectionnés par le peintre. Mais ici les couleurs sont vives à l'intérieur de l'établissement qui ressemble à un aquarium. Le rouge, le vert et le jaune font des contrastes qui ne sont pas sans rappeler le Café de nuit ou la Terrasse du café la nuit de Van Gogh. Les quatre personnages sont immobiles. La femme et l'homme sont côte à côte, mais on ne sait ce que tient dans sa main cette femme rousse vêtue d'une robe rouge : est-ce un sandwich, un paquet de cigarettes, une liasse de billets? Est-elle une prostituée? Que veut dire cette caisse enregistreuse qui trône, étrange seule, dans la vitrine de l'autre côté de la rue et qui évoque la marchandisation de la société capitaliste? Le couple trouve en quelque sorte 
son double dans les deux percolateurs derrière le barman, image de machine sans vie faisant écho à la morne apathie des deux personnages.

Ils sont là perdus dans la nuit, dans leur nuit, avec leurs songes dérisoires et leur insomnie trompée à coups de tasses de café exécrable [...] Ils sont profondément seuls. Chacun pense à son propre rêve, à son propre destin. Ils assument leur solitude depuis si longtemps qu'elle n'a plus rien de désespérés. adaptée au cinéma en 1946 par Robert Siodmak et qui narre l'histoire de deux tueurs à gages qui attendent leur victime, un ex-boxeur, dans une brasserie. Aussi le mal-être est-il lié à l'idée de la mort, peut-être également au poème de W.H. Auden, « September I, 1939 » qu'il appréciait et qui évoquait un monde sans défense figé dans la stupeur sous la nuit. Chez Hemingway, les tueurs après s'en être pris au serveur, au cuisinier et à Nick Adams attendent, pour le tuer, ole Andreson qui fréquente régulièrement le restaurant. Mais ce soir-là, le boxeur suédois ne vient pas. Le serveur envoie Nick, après le départ des tueurs désappointés, pour prévenir Andreson. Mais celui-ci ne réagit pas. Est-il indifférent, défaitiste? On ne le sait. Aussi finit-il, devant l'apathie de tous, à quitter la ville. Les oiseaux de nuit sont peut-être les deux tueurs en chômage ou attendant leur heure. Le visage de l'homme près de la femme, au fort nez marqué, légitime l'appellation d'oiseau de nuit (Nighthawks), perché un peu en avant sur le comptoir. Quant à l'autre personnage portant un chapeau identique au premier, il est encore plus mystérieux, nous tournant le dos, tenant pensivement son verre à la main.

Solitude, enfermement, caractérisent ces noctambules renfermés dans cette insondable intimité, sans doute vide, à l'instar des fenêtres obscures qui dominent et équilibrent en le rythmant le tableau. Le bar ou le café sont les derniers lieux de refuge pour ces êtres chassés par la nuit et s'accrochant au comptoir (comme dans le poème d'Auden, "Children afraid of the night/Who have never been happy or good»). Car l'obscurité est menaçante comme on le voit par exemple dans Night in the Park et Hopper multiple les images de lieux pouvant servir de réconfort, comme par exemple avec Rooms for Tourists ou encore avec l'huile de 1940 Gas (en fait crépuscule, mais Hopper, avait dit qu'il voulait peindre "l'effet de la nuit sur une station service»), station-service qui sert de refuge contre la nuit avec ses lumières, offrant de l'essence jour et nuit, pour continuer, pour ne pas être en panne, donnant l'assurance d'un lien, sans doute anonyme, lieu de secours mais troublant, étrangement familier sans l'être cependant (c'est là la définition de l'Unheimlich). On a pu voir ici une représentation de l'aliénation américaine, celle de l'homme de la classe moyenne, ici asservi par la technique qu'il sert comme un prêtre servirait un dieu ou des idoles (elles sont trois! trois pompes à essence), aliénation dévote et quasi religieuse et mythique comme en témoigne l'image de Pégase, mais ramenée à une figure publicitaire incarnant un grand groupe pétrolier.

Richard Sennett a, dans son ouvrage The Fall of Public Man, décrit le déséquilibre de la vie privée et $\mathrm{du}$ vide de la sphère publique dans un monde voué à l'anonymat, l'aliénation et la froideur. Il est vrai que les personnages isolés les uns des autres dans les tableaux de Hopper n'ont pas besoin d'une cage de verre comme dans les Oiseaux de nuit. Car la solitude des grandes villes et la mort de l'espace public amènent une destructivité involontaire, celle du "paradoxe de l'isolement dans la transparence » suivant Richard Sennett.

L'huile de 1927, intitulée significativement Automat, montre que la femme est de passage, elle n'a retiré qu'un seul de ses gants et a gardé son manteau (à moins que la 
solitude, renforcée par la chaise vide en face d'elle, ne la frigorifie en dépit du radiateur à gauche, qui, comme objet technique, ne sert à rien pour réchauffer l'âme). Le repli intérieur est mis en relief par le miroir vide derrière elle. De fait, pour les critiques, il s'agit d'une grande vitre reflétant l'éclairage intérieur et l'extérieur est entièrement barré par l'obscurité de la nuit. Mais cette vitre/miroir ne reflète pas la passante ${ }^{52}$.

Dans Compartiment $C$, voiture 193 (1938) le monde extérieur disparaît, plongé dans la nuit qui tombe, la femme dont le chapeau masque le regard est absorbée dans sa lecture illustrant cette "tyrannie de l'intimité » que décrit Richard Sennett. Comme si la nuit renfermait les êtres en eux-mêmes, les scènes de la vie nocturne stigmatisent l'incommunicabilité.

Une maison éclairée dans la nuit a fasciné tous ceux qui, perdus dans la vaste obscurité, cherchent un refuge, un abri, un lieu intime et réconfortant. Mais le motif de la fenêtre d'où transparaît une lumière qui tranche avec le noir est également le ressort d'une curiosité, celle de connaître ce qui se trame dans l'intimité des gens. Baudelaire a magistralement décrit dans le poème en prose Les Fenêtres (dans Le Spleen de Paris) la fascination exercée sur l'imagination et le pouvoir de la fiction spéculant sur l'intimité de la vie des gens.

Celui qui regarde du dehors à travers une fenêtre ouverte, ne voit jamais autant de choses que celui qui regarde une fenêtre fermée. Il n'est pas d'objet plus profond, plus mystérieux, plus fécond, plus ténébreux, plus éblouissant qu'une fenêtre éclairée d'une chandelle. Ce qu'on peut voir au soleil est toujours moins intéressant que ce qui se passe derrière une vitre. Dans ce trou noir ou lumineux vit la vie, rêve la vie, souffre la vie ${ }^{53}$.

Barbey d'Aurevilly, lui aussi, dans le Rideau cramoisi des Diaboliques, non sans se souvenir de Baudelaire, a décrit la rêverie du narrateur qui s'interroge sur ce qu'il pouvait y avoir derrière les rideaux d'une fenêtre la nuit. Car dans la ville endormie une fenêtre éclairée témoignant de la veille d'un être humain à une heure avancée alors que tout le monde dort, a quelque chose d'étrange et d'imposant.

Mais l'ignorance de ce qui fait veiller derrière une fenêtre aux rideaux baissés, où la lumière indique la vie et la pensée, ajoute la poésie du rêve à la poésie de la réalité. Du moins, pour moi, je n'ai jamais pu voir une fenêtre, - éclairée la nuit, - dans une ville couchée, par laquelle je passais, sans accrocher à ce cadre de lumière un monde de pensées, sans imaginer derrière ces rideaux des intimités et des drames... 54

61 La lumière tamisée par «le double rideau cramoisi dont elle traversait mystérieusement l'épaisseur ${ }^{55}$ » invite à imaginer l'intimité mystérieuse et inconnue se cachant derrière celui, qui voile par l'opacité de sa couleur le mystère qu'il dérobe aux regards. C'est bien une présence de la vie intime que révèle une telle lumière au milieu de l'ombre environnante. Mais cette intimité échappe doublement, car dans le récit que vous connaissez, l'amante - tout comme le "carré vide, rouge et lumineux ${ }^{56}$ » du fameux rideau - reste énigmatique. Alberte est vide de sens, tout comme la fenêtre est vide de tout signe déchiffrable. Car lors des visites nocturnes d'Alberte dans l'espace clos de la chambre de Brassard, non seulement le rideau est baissé « afin que les voisins, plus curieux en province qu'ailleurs, ne dévisageassent pas le fond de [sa] chambre ${ }^{57}$ ", mais Alberte elle-même est repliée sur elle-même, froide comme un marbre impénétrable. Aussi l'amante n'a-t-elle jamais dévoilé sa vie intime (à supposer qu'elle en eût une) et elle est restée aussi obscure et indéchiffrable que la nuit elle-même. 
62 On retrouve chez Proust, dans $\mathrm{Du}$ côté de chez Swann la même fascination pour cette nitescence convoquant un regard voyeuriste pour pénétrer, en vain, dans une intimité à jamais inaccessible. Swann, doutant de la fidélité d'Odette qu'il venait de quitter, était retourné dans son quartier où « tout était désert et noir » pour espionner la fenêtre en essayant de deviner ce qui se passe dans la chambre :

Parmi l'obscurité de toutes les fenêtres éteintes depuis longtemps dans la rue, il en vit une seule d'où débordait - entre les volets qui en pressaient la pulpe mystérieuse et dorée - la lumière qui remplissait la chambre et qui, tant d'autres soirs, du plus loin qu'il l'apercevait, en arrivant dans la rue, le réjouissait et lui annonçait : « elle est là qui t'attend » et qui maintenant, le torturait en lui disant : " elle est là avec celui qu'elle attendait ». Il voulait savoir qui ; il se glissa le long du mur jusqu'à la fenêtre, mais entre les lames obliques des volets il ne pouvait rien voir; il entendait seulement dans le silence de la nuit le murmure d'une conversation $^{58}$.

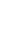
yorkais, qui ne manquèrent pas d'en exploiter les ressources visuelles ${ }^{59}$ ». On a déjà vu que Hopper lui a consacré plusieurs tableaux. Il fut inspiré en cela par son maître John Sloan à qui il a emprunté le titre de Night Windows. Dans ce tableau, Sloan, isolant des scènes mettant à nu la vie privée des gens, on aperçoit une femme en train de se coiffer, les bras levés et une autre étendant son linge, les deux attitudes mettant en évidence la sensualité de leurs corps. Un homme sur le toit joue le rôle du voyeur de cette scène réaliste, caractéristique du réalisme de l'Ash Can School. 


\section{BIBLIOGRAPHIE}

Banu, Georges. Nocturnes. Peindre la nuit. Jouer dans le noir. Paris : Biro, 2005.

Baudelaire, Charles. Euvres Complètes. Paris : Gallimard, Bibliothèque de la Pléiade, 1975.

Cabantous, Alain. Histoire de la nuit, XVIIe-XVIIIe siècle. Paris : Fayard, 2009.

Capote, Truman. Un arbre de nuit et autres histoires. (traduction de S. Doubrovsky), Paris :

Gallimard, 1998.

Delattre, Simone. Les douze heures noires. La nuit à Paris au XIX siècle. Paris : Albin Michel, 2003.

Freud, Sigmund. L'inquiétante étrangeté et autres essais. Paris : Gallimard, 1985.

Gautier, Théophile. Mademoiselle de Maupin, in Euvres Complètes. Paris : Honoré Champion, 2004.

Guérin, Floran (ed.). Cohabiter les nuits urbaines. Paris : L'Harmattan, 2018.

Liszt, Franz. Gesammelte Schriften. Hildesheim- New York: Georg Olms Verlag, 1978. 
Montandon, Alain (ed.), Dictionnaire littéraire de la nuit. Paris: Honoré Champion, 2013.

Schlör, Joahim. Nachts in der großen Stadt. Paris, Berlin, London 1840-1930. München : Artemis und Winkler, 1991 (Nights in the Big City. London : Reaktion, 1998).

Valance, Hélène. Nuits américaines. L'art du nocturne aux USA. 1890-1917. Paris, Sorbonne Université Presses, 2015.

\section{NOTES}

1. Dans sa préface à l'édition de "Neuf nocturnes » de Field (Liszt, Franz. Gesammelte Schriften. Hildesheim- New York: Georg Olms Verlag, 1978, IV, p. 263-271.)

2. Baudelaire, Charles. "Recueillement " (1861) in Les Fleurs du mal. CEuvres complètes. [édition posthume 1868], Paris : Gallimard, Bibliothèque de la Pléiade, 1975, I, p. 141.

3. Gautier, Théophile. Mademoiselle de Maupin, in Oeuvres Complètes. Paris : Honoré Champion, 2004, I, 1, p. 191-192.

4. Mann und Frau den Mond betrachtend, huile sur toile, 34x44, 1830-1835, Nationalgalerie Berlin.

5. Novalis, Le Brouillon général, $\mathrm{n}^{\circ} 342$, in Schriften. Wissenschlaftliche Buchgesellschaft, Darmstadt, 1968, vol. III, p. 302.

6. Jullien, François. De l'intime : loin du bruyant amour. Paris : Grasset, 2013.

7. «La rue éclairée fait silence », Hölderlin, Friedrich. Euvre poétique complète. Traduction de François Garrigue, Paris, Éditions de la Différence, 2005, p. 693.

8. «Calmes dans l'air assombri tintent les cloches...», Hölderlin, Friedrich. Euvre poétique complète. Op. cit., p. 695.

9. Novalis. Grains de pollen, $\mathrm{n}^{\circ} 24$, in Schriften. Wissenschlaftliche Buchgesellschaft, Darmstadt, 1965, vol. II, p. 423.

10. Comme chez Baudelaire dans Les Fleurs du mal: "Vous êtes tous les deux ténébreux et discrets : / Homme, nul n'a sondé le fond de tes abîmes ; / Ô mer, nul ne connaît tes richesses intimes ».

11. Schlör, Joahim. Nachts in der großen Stadt. Paris, Berlin, London 1840-1930. München : Artemis und Winkler, 1991 (Nights in the big City. London : Reaktion, 1998).

12. «Que d'autres peignent ce qui arrive le jour ; moi, je vais crayonner les iniquités nocturnes... J'ai vu ce que personne que moi, n'a vu.» (Restif de la Bretonne. Les Nuits de Paris, Première Nuit. In : Paris le jour, Paris la nuit. Paris, Robert Laffont, 1990, p. 620).

13. Janin, Jules. L'Artiste. Article « Le Daguerotype », janvier 1839, p. 148.

14. Delattre, Simone. Les douze heures noires. La nuit à Paris au XIXe siècle. Paris : Albin Michel, 2003, p. 97.

15. Vallès, Jules. Le Tableau de Paris, in CEuvres complètes. Paris : Editeurs Français réunis, 1971, p. 54.

16. Baudelaire, Charles. "Le cygne ", Tableaux parisiens, in Euvres complètes. Paris : Gallimard, Bibliothèque de la Pléiade, 1975, I, p. 85.

17. Zola, Émile. Les Rougon-Macquart : Histoire naturelle et sociale d'une famille sous le Second Empire. éd. intégrale publiée sous la dir. d'Armand Lanoux, études, notes et variantes par Henri Mitterand, Paris : Gallimard, coll. Bibliothèque de la Pléiade, t. I, 1960, p. 453.

18. Rattier, Paul-Ernest de. Paris n'existe pas. Paris, Bordeaux : Imp. de Balarac jeune, 1857.

19. Janin, Jules. Le Livre des Cent-et-Un. Paris : Ladocat, 1833, tome 10, p. 57.

20. Walter Benjamin cite dans Capitale du XIXe siècle, Paris : Editions du Cerf, 1993, p. 457, à propos de L'homme des foules d'Edgar A. Poe un texte extrait d'un article de La Semaine du 4 octobre 1846, attribué à Balzac ou à Hippolyte Castille : "L'œil s'attache à cet homme qui marche dans la 
société à travers les lois, les embûches, les trahisons de ses complices, comme un sauvage du nouveau monde parmi les reptiles, les bêtes féroces et les peuplades ennemies. »

21. Banu, Georges. Nocturnes. Peindre la nuit. Jouer dans le noir. Paris : Biro, 2005.

22. Banville connaît lui aussi l'âme du noctambule quand il écrit: "Il y a eu alors des Noctambules qui regardaient et adoraient la Ville comme une maîtresse endormie, et c'était leur joie, leur volupté et leur ravissement de marcher en contemplant le ciel parisien, et d'en jouir comme d'une chose qui leur appartient en propre [...]», Banville, Théodore de. Petites études, l'âme de Paris : Nouveaux souvenirs. 1890 p. 290.

23. Maupassant, Guy de. Contes et Nouvelles. Paris : Robert Laffont, Bouquins, 1988, vol. I, p. 599.

24. Ibid., p. 600.

25. Baudelaire, Charles. "Le Crépuscule du soir », Le Spleen de Paris, XXII, in : CEuvres Complètes, Bibliothèque de la Pléiade, 1975, t. I, p. 311.

26. C'est là un thème quasiment obligé qui, de Restif de la Bretonne à Lautréamont, ne cesse d'être évoqué à la tombée de la nuit (l'on sait que sa compagne, la chouette, depuis fort longtemps prend également son vol).

27. Maupassant, Guy de. Contes et Nouvelles. Op. cit., p. 601.

28. Ibid.

29. Ibid., p. 603.

30. Bancquart, Marie-Claire. Images littéraires du Paris "fin-de-siècle». Paris: Editions de la Différence, 1979, p. 153.

31. Deschamps, Catherine. "Le genre du Droit à la nuit parisienne ", in Guérin, Floran (éd.). Cohabiter les nuits urbaines. Paris : L'Harmattan, 2018, p. 32.

32. Freud, Sigmund. L'inquiétante étrangeté et autres essais. Paris : Gallimard, 1985, p. 263.

33. Proust, Marcel. A la Recherche du temps perdu. Paris : Gallimard, Pléiade, t. 1, 1987, p. 28.

34. Bachelard, Gaston. L'eau et les rêves. L'imagination de la matière. Paris: Librairie Générale Française, 1993, p. 122.

35. Cabantous, Alain. Histoire de la nuit, XVIIe-XVIIIe siècle. Paris : Fayard, 2009, p. 42.

36. Montandon, Alain (Dir.). Dictionnaire littéraire de la nuit. Paris: Honoré Champion, 2013, p. 1027-1039.

37. Capote, Truman. Un arbre de nuit et autres histoires. Paris: Gallimard, traduction de Serge Doubrovsky et Maurice Edgar Coindreau, 1998, p. 209.

38. C'est " comme si ce train venait de nulle part et conduisait ses étranges voyageurs vers un endroit improbable », Cassirame, Brigitte. Les visages de la mélancolie. Volume 3, Paris : Publibook, 2010, p. 13.

39. Cassirame, Brigitte. Les visages de la mélancolie. Op. cit., p. 26.

40. Capote, Truman. Un arbre de nuit et autres histoires. Op. cit., p. 210.

41. Ibid., p. 215.

42. Une formation dévalorisée par la femme qui rétorque à Kay: "Qu'est-ce que tu veux donc apprendre dans un endroit pareil? Laisse moi te dire, mon chou, que pour ce qui est de l'éducation, j'en ai pas qu'un peu, et pourtant, j'ai jamais mis les pieds dans une université ! » (Ibid., p. 212).

43. Ibid., p. 227.

44. Voir Montandon, Alain. Mélusine et Barbe-Bleue. Paris : Éditions Champion, 2018.

45. «Il le serre bien, il lui tient chaud. » (Erlkönig, in Deutsche Balladen,München, Goldman, 1961, p. 22).

46. Id.

47. Capote, Truman. Un arbre de nuit et autres histoires. Op. cit., p. 228.

48. Capote ,Truman. Un arbre de nuit et autres histoires. Op. cit.

49. Capote, Truman. Un arbre de nuit et autres histoires. Op. cit.., p. 226.

50. Cassirame, Brigitte. Les visages de la mélancolie. Op. cit., p. 34. 
51. Polanski, Roman. «Quand la ville dort ». Le Nouvel Observateur, 18 mai 1984.

52. «The painting suggests several things, but the most obvious and most resonant is that if what the window reflects is true then the scene takes place in limbo and the seated woman is an illusion. This is a troubling idea. And if the woman thinks of herself in this context, she cannot possibly be happy. But of course she does not think, she is the product of another will, an illusion, an invention of Hopper's. ", Strand, Mark. Hopper. New York: Knopf, 2007, p. 43.

53. Baudelaire, Charles. Les Fenêtres dans Le Spleen de Paris. CEuvres Complètes. Paris : Gallimard, Bibliothèque de la Pléiade, 1975, t. I, p. 339.

54. Barbey d'Aurevilly, Jules. "Le Rideau cramoisi », Les Diaboliques, in CEuvres romanesques complètes. Paris : Gallimard, Bibliothèque de la Pléiade, t. II, 1966, p. 19-20.

55. Ibid., p. 21.

56. Ibid., p. 57.

57. Ibid., p. 42.

58. Proust, Marcel. Du côté de chez Swann in À la recherche du temps perdu. Paris: Gallimard, Bibliothèque de la Pléiade, 1954, t. I, p. 272-273.

59. Valance, Hélène. Nuits américaines. L'art du nocturne aux USA. 1890-1917. Paris: Sorbonne Université Presses, 2015.

\section{RÉSUMÉS}

La nuit porte en elle le langage de l'inexprimable. Harmonieuse, maternelle, profonde, elle ouvre aussi les portes de l'abîme. Forçant à plonger dans l'inconnu, elle en expose les affres et les tourments. Les fenêtres répètent cette incommunicabilité qu'elle révèle envers les autres comme envers soi.

\section{INDEX}

Keywords : intimacy, night, nocturnal, harmony, conversion, unconscious, decline, window, inscrutability, identity, city

Mots-clés : intimité, nuit, nocturne, harmonie, conversion, inconscient, régression, fenêtre, impénétrabilité, identité, ville

\section{AUTEURS}

\section{ALAIN MONTANDON}

Professeur émérite

Celis

alain.montandon@uca.fr 